\title{
La rúbrica como instrumento de evaluación de la competencia de indagación científica
}

The rubric as an instrument for evaluating the competence of

scientific inquiry

Recepción del artículo: 15-11-2020 ～Aceptación del artículo: 29-12-2020

Nelly Dorita, Tuesta Calderón

Universidad Nacional de Educación Enrique Guzmán y Valle nellydorita@hotmail.com

\section{https://orcid.org/0000-0001-9709-1196}

Para referenciar este artículo:

Tuesta-Calderón, N. D. (2021). La rúbrica como instrumento de evaluación de la competencia de indagación científica. Revista ConCiencia EPG, 6(1), 24-35. https://doi.org/10.32654/CONCIENCIAEP

G.6-1.2

\section{Resumen}

e presenta una investigación cuyo propósito fue demostrar si el uso de rúbricas de evaluación mejora el nivel de desempeño de la competencia de indagación científica. Para ello, se diseñó un estudio cuasi experimental en la cual participaron 42 estudiantes del tercer año de educación secundaria de la Institución Educativa "Santo Toribio de Mogrovejo"-Zaña, 21 para grupo control y 21 para grupo experimental. La evaluación del pretest y postest se realizó mediante un examen escrito con diez ítems, con alternativas que contenían niveles de logro de capacidades desde inicio hasta logro destacado. Luego de ello, se aplicó al grupo experimental una guía de actividades indagatorias con sesiones de clase que buscaron fortalecer cada una de las capacidades de la competencia de indagación, y estas se fueron evaluadas mediante una rúbrica al final de la unidad. En los resultados se evidenció que no existe diferencias significativas entre las notas de los estudiantes que se aplicó las rubricas, con los que no se aplicó las rubricas para la evaluación de la competencia de indagación científica. Por lo que se concluye que la utilización de la rúbrica en la evaluación no influye de manera significativa en el desarrollo de las capacidades de la competencia de indagación científica.

Palabras Clave: Rúbricas; indagación científica; capacidades, desempeños; competencia. 
Abstract

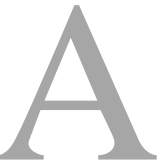

$\mathrm{n}$ investigation is presented whose purpose was to demonstrate if the use of evaluation rubrics improves the level of performance of the scientific inquiry competence, for this, a quasiexperimental study was designed in which 42 students of the third year of secondary education of the Educational Institution "Santo Toribio de Mogrovejo" -Zaña, 21 for control group and 21 for experimental group. The pre-test and post-test evaluation were carried out by means of a written exam with ten items, with alternatives that contained levels of achievement of abilities from beginning to outstanding achievement. After that, an investigative activities guide was applied to the experimental group with class sessions that sought to strengthen each of the capacities of the inquiry competence, and these were evaluated through a rubric at the end of the unit. The results show that there are no significant differences between the grades of the students who applied the rubrics, with those who did not apply the rubrics, regarding the development of scientific inquiry competence. Therefore, it allows to conclude that the use of the rubric in the evaluation does not significantly influence the development of the capacities of the scientific inquiry competence.

Key Words: Rubrics; scientific inquiry; capabilities, performances; competition.

\section{Introducción}

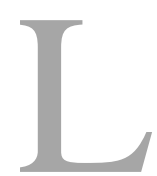

a indagación científica teóricamente se sustenta en el enfoque de la complejidad en el proceso de enseñanza (Mesta, 2018), para ello, se trata de preparar a los docentes en sólidos dominios de contenidos con una cuota importante de estrategias didácticas para la enseñanza efectiva (López, 2017), debido a que esto genera cambios conceptuales y argumentativos en los estudiantes (Camacho, Casilla \& Finol, 2008), según el Currículo Nacional (2016), implica que los estudiantes combinen una serie de capacidades como: problematiza situaciones, diseña estrategias para hacer indagación, genera y registra datos o información, analiza datos e información, evalúa y comunica el proceso y resultados de su indagación. Más concretamente, la estrategia de indagación es concebido como el instrumento y procedimiento utilizado por los docentes y estudiantes para construir o reconstruir el aprendizaje, es una ruta procedimental en el aprendizaje de la investigación en un aula de clase (Camacho, Casilla \& Finol, 2008), en las que sirven como actividades estudiantiles para desarrollar conocimientos y entendimiento de las ideas científicas (Schwab,1960; 1966; 1978). Sin embargo, esto no solo le sirve a los estudiantes sino, que los docentes también logran avanzar de manera satisfactoria en el aprendizaje y la enseñanza de las ciencias (Cristobal y García, 2013; González, Cortéz, Bravo, Ibaceta, Cuevas, Quiñones, Maturana \& Abarca, 2012).

El problema es que, en el sistema educativo actual, no se aplica este enfoque, 
y los docentes desconocen el marco teórico y el uso de la metodología indagatoria para enseñar ciencias a sus estudiantes (Cristobal y García, 2013) y estos no logran dar una razón argumentativa, tolerante, consensual, plural, reflexiva y analítica sobre un objeto de estudio (Márquez, 2016), se desconoce los procesos y los instrumentos de aprendizaje como es la rúbrica para ir construyendo y evaluando este proceso.

Una de las principales causas es que, tradicionalmente este tipo de educación y formación de los estudiantes en educación básica ha girado en torno a una enseñanza desagregada del saber científico (Vilches, 2001), está enfocada en la acumulación de información irrelevante para la vida actual, en lugar de desarrollar actitudes y habilidades necesarias para el aprendizaje y la practica científica (National Research Council. NCR, 2000). Además, es una enseñanza descontextualizada de las necesidades y problemáticas sociales actuales, de la vida y problemáticas cotidianas de las personas (Albertini et al., 2005; Chacón 2003; Pasmanik et al., 2005). Otra causa del problema es que en nuestro país como en otros lugares se tiene escasa cultura científica (Domínguez, 2012), pero también, un factor importante es que en nuestro medio no hay políticas educativas ni científicas claras como para comprometer a los niños en esas tareas y lleva a los jóvenes a pensar en la ciencia como algo no reditable 0 atractivo (Pelcastre, Gómez \& Zavala, 2015).

Dentro de las consecuencias que ha generado esta problemática, es que, los estudiantes que pasan a la educación superior tienen grandes dificultades para hacer investigación. Según sostiene Vilches (2001) la educación secundaria es la etapa en donde se debería aprender la alfabetización científica. Sin embargo, de acuerdo con los datos de Hernández (2015) la enseñanza de la ciencia en esta etapa de educación es la menos favorable, esto tendría que ver con las deficiencias que los mismos profesores tienen para identificar implicancias sociales de la ciencia y la tecnología (Acevedo et al., 2002, 2003, 2005). Y otra consecuencia y seguramente la más importante es que no se ha desarrollado algunos componentes esenciales en los estudiantes que tienen que ver con el proceso de enseñanzaaprendizaje. Bybee (2004) señala que cuando existe una enseñanza basado en la indagación se desarrollan tres componentes: la primera, tiene que ver con las habilidades de indagación, lo que está dentro de la obligación de los estudiantes y no lo hacen. El segundo componente es el conocimiento sobre la indagación misma, lo que deben comprender los estudiantes sobre la importancia y la naturaleza de la indagación en su formación. Y el tercero, es desarrollar una aproximación pedagógica para la enseñanza de los contenidos científicos en cada materia, esto estaría relacionado con la labor del docente.

Así mismo, dentro del sistema educativo, según refiere Martin-Hansen (2002) también existen varios tipos indagación. La primera es la indagación abierta. Se centra en el estudiante, quien intenta responder una pregunta para lo cual diseña un plan de una investigación o experimento $y$ finalmente realiza la comunicación de los resultados. El segundo 
tipo es la indagación guiada. Está a cargo del docente que guía y orienta a los estudiantes en el proceso de indagación en el aula de clase o en un laboratorio. El tercer tipo es la indagación acoplada. En el proceso utiliza las acciones de las dos primeras, es decir acopla tanto la indagación abierta como guiada. Y el cuarto tipo, es la indagación estructurada. Es un proceso minucioso dirigida por el docente en donde enseña a los estudiantes procesos específicos para obtener datos $\mathrm{y}$, lograr objetivos específicos.

Como se ha descrito, existen en nuestro medio grandes dificultades por la que los estudiantes no desarrollan competencias de indagación, he ahí la importancia del estudio y del programa que consiste en aplicar un conjunto de módulos sobre indagación científica en los estudiantes, el mismo que también puede servir como una forma de innovar la enseñanza de los docentes, dado que, según comenta González, Cortéz, Bravo, Ibaceta, Cuevas, Quiñones, Maturana \& Abarca (2012), una manera innovadora de concebir la enseñanza de las ciencias, es justamente, aprehendiendo el concepto de indagación científica y desarrollado de manera adecuada en los estudiantes.

Estas capacidades se pueden desarrollar y evaluar a través de sesiones de aprendizaje orientadas a la indagación o investigación experimental de los estudiantes. Principalmente, con la finalidad de poder evaluar los niveles de desempeño de esta competencia se utiliza la rúbrica de evaluación que es un" registro evaluativo que posee ciertos criterios o dimensiones a evaluar y lo hace siguiendo unos niveles o gradaciones de calidad y tipificando los estándares de desempeño" Cano (2015). Permite al profesor especificar claramente lo que espera de los estudiantes, en cuanto a su aprendizaje y cuáles son los criterios con que se calificará un determinado trabajo o actividad, ya sea una presentación oral o un reporte (Ahumada, 2005).

La rúbrica constituye un instrumento que coadyuva en el aprendizaje de competencias de los estudiantes (Raposo y Martínez, 2014) y también es una buena opción para evaluar un informe de laboratorio, un ensayo original, un prototipo o modelo (Díaz Barriga, 2005). Por ello, la importancia del trabajo es aportar al conocimiento existente más información sobre el uso de las rúbricas como instrumento de evaluación de la competencia de indagación científica en la educación secundaria. En ese sentido, el propósito del estudio fue analizar la importancia de la rúbrica en el desarrollo de la indagación científica en estudiantes de educación secundaria.

\section{Método}

Diseño. El estudio se desarrolló bajo un diseño cuasi-experimental con dos grupos, un grupo de control y uno de experimento.

Participantes. La población de estudio estuvo compuesta por un total de 65 participantes, tres secciones del tercer grado de educación secundaria, dos secciones que estudian en el turno de la mañana y una en la tarde, en la Institución Educativa "Santo Toribio de Mogrovejo"- 
Zaña, un distrito urbano-rural de la provincia de Chiclayo, región Lambayeque. La muestra fue de 42 estudiantes de sexo femenino, cuyas edades fluctúan entre 14 y 15 años del tercer grado de educación secundaria, que estudian en el turno de la mañana y fueron seleccionados por muestreo probabilístico, porque todos tuvieron la posibilitad de ser seleccionados como muestra.

Instrumento. El instrumento que se utilizó para evaluar la competencia de indagación científica en los estudiantes fue la rúbrica analítica. Esta rúbrica posee cuatro componentes específicos que evalúan los procesos de desarrollo de esta competencia que va desde 0 a 10 (en inicio); de 11 a 13 (en proceso); de 14 a 17 (logro esperado) y, de 18 a 20 (logro destacado).

Procedimiento. Al principio se diseñaron los módulos para trabajar las competencias de indagación científica que fueron evaluadas mediante las rúbricas. Luego se le comunicó al director y a los padres de familia sobre el objetivo del estudio, para que den el permiso correspondiente e iniciar el estudio. Una vez conseguido el permiso se eligió las secciones para el grupo control y grupo experimental con lo que se inició el proceso de recojo de datos, primero la evaluación del pretest a ambos grupos, luego la aplicación del módulo al grupo experimental que tuvo una duración de cuatro meses. Una vez concluido la aplicación del módulo se realizó la evaluación del postest para ambos grupos con lo cual se hizo el análisis estadístico utilizando el software SPSS en su versión 23.

\section{Resultados}

Dentro de este apartado se presentan los resultados del estudio tanto descriptivos como inferenciales, a nivel descriptivo se presentan los resultados de pretest del grupo control y grupo experimental y luego el resultado del postest de ambos grupos, posteriormente se presentan los resultados inferenciales, es decir, la comparación de ambos grupos en el pretest y postest.

\section{Tabla 1}

Prueba pretest- grupo control y grupo experimental

\begin{tabular}{llcccc}
\hline ítems & grupos & 0 a 10 & $\mathbf{1 1}$ a 13 & $\mathbf{1 4}$ a 17 & $\mathbf{1 8}$ a 20 \\
\hline $\begin{array}{l}\text { Competencia de indaga } \\
\text { mediante métodos científicos }\end{array}$ & Control & $67 \%$ & $19 \%$ & $14 \%$ & $0 \%$ \\
$\begin{array}{l}\text { para construir sus } \\
\text { conocimientos. }\end{array}$ & Experimental & $62 \%$ & $14 \%$ & $19 \%$ & $5 \%$ \\
\hline Problematiza situaciones. & Control & $67 \%$ & $0 \%$ & $0 \%$ & $33 \%$ \\
& Experimental & $67 \%$ & $0 \%$ & $0 \%$ & $33 \%$ \\
\hline
\end{tabular}




\begin{tabular}{llllll}
\hline Diseña estrategias de & Control & $86 \%$ & $0 \%$ & $0 \%$ & $14 \%$ \\
indagación. & Experimental & $86 \%$ & $0 \%$ & $0 \%$ & $14 \%$ \\
\hline Registra datos e información. & Control & $71 \%$ & $0 \%$ & $0 \%$ & $29 \%$ \\
& Experimental & $76 \%$ & $0 \%$ & $0 \%$ & $24 \%$ \\
\hline Analiza datos e información. & Control & $76 \%$ & $0 \%$ & $0 \%$ & $24 \%$ \\
& Experimental & $81 \%$ & $0 \%$ & $0 \%$ & $19 \%$ \\
\hline Evalúa y comunica los & Control & $86 \%$ & $0 \%$ & $0 \%$ & $14 \%$ \\
resultados de su indagación. & Experimental & $57 \%$ & $0 \%$ & $0 \%$ & $43 \%$ \\
\hline
\end{tabular}

En la tabla 1 se puede apreciar que la mayoría de los estudiantes en el pretest obtuvieron un promedio bajo en cuanto a las dimensiones evaluadas, por lo que se puede mencionar que se considera que los estudiantes, antes del inicio de la aplicación de los módulos, no tienen noción sobre la importancia de las competencias para el aprendizaje de los temas desarrollados por el docente, lo cual es un indicador, de que el estudiante está en un nivel inicial de desempeño.

Tabla 2

Prueba postest- grupo de control y grupo experimental

\begin{tabular}{llcccc}
\hline Ítems & Grupos & 0 a 10 & 11 a 13 & $\mathbf{1 4}$ a 17 & $\mathbf{1 8}$ a 20 \\
\hline Competencia de indaga & Control & $57 \%$ & $14 \%$ & $29 \%$ & $0 \%$ \\
mediante métodos científicos, & Experimental & $43 \%$ & $14 \%$ & $29 \%$ & $14 \%$ \\
para construir sus & & & & & \\
conocimientos. & & & & \\
Problematiza situaciones. & Control & $67 \%$ & $0 \%$ & $0 \%$ & $33 \%$ \\
& Experimental & $67 \%$ & $0 \%$ & $0 \%$ & $33 \%$ \\
Diseña estrategias de & Control & $48 \%$ & $0 \%$ & $0 \%$ & $48 \%$ \\
indagación. & Experimental & $52 \%$ & $0 \%$ & $0 \%$ & $48 \%$ \\
Registra datos e información. & Control & $62 \%$ & $0 \%$ & $0 \%$ & $38 \%$ \\
& Experimental & $57 \%$ & $0 \%$ & $0 \%$ & $43 \%$ \\
Analiza datos e información. & Control & $95 \%$ & $0 \%$ & $0 \%$ & $5 \%$ \\
& Experimental & $95 \%$ & $0 \%$ & $0 \%$ & $5 \%$ \\
Evalúa y comunica los & Control & $62 \%$ & $0 \%$ & $0 \%$ & $38 \%$ \\
resultados de su indagación. & Experimental & $52 \%$ & $0 \%$ & $0 \%$ & $48 \%$ \\
\hline
\end{tabular}


En esta tabla 2, se presentan los resultados del postest de ambos grupos en donde se evidencia que los estudiantes, en su mayoría se encuentran aún en el nivel inicial de desempeño, por más que al grupo experimental se les haya proporcionado un módulo adicional para desarrollar las competencias de indagación científica, éstos no logran avanzar de manera significativa en las capacidades de competencia de indagación científica. Si bien, hay un avance con respecto al pretest, el porcentaje de estudiantes sigue siendo alto en el nivel inicial a diferencia de los otros niveles que no superan el $50 \%$ de avance.

\section{Tabla 3}

Comparación de medias de pretest y postest grupo control y grupo experimental

\begin{tabular}{lcc}
\hline & Pretest & Postest \\
\hline Grupo control & 9,33 & 10,29 \\
Grupo experimental & 10,48 & 12,19 \\
\hline
\end{tabular}

De acuerdo con las medias del pretest y postest, en la tabla 3, se evidencia que hay pequeñas diferencias antes y después de la aplicación del módulo para desarrollar competencias de indagación científica, estas diferencias no son significativas por lo que se concluye que, la competencia de indagación científica en los estudiantes de educación básica es un proceso que necesita ser aplicado durante periodos más extensos o, grados de estudio completo.

\section{Tabla 4}

Comparación de medias de las dimensiones entre grupos

\begin{tabular}{|c|c|c|c|c|c|c|c|c|c|}
\hline Competencias & Grupos & Media & $\boldsymbol{\sigma}$ & $\begin{array}{l}\mathrm{N}^{\circ} \\
\text { de } \\
\text { obs. }\end{array}$ & $\sigma^{2}$ & gl & $\mathbf{t}$ & $\begin{array}{l}P \\
(T<=t) \\
\text { dos } \\
\text { colas }\end{array}$ & $\begin{array}{l}\text { Valor } \\
\text { crítico de } \\
\text { t (dos } \\
\text { colas) }\end{array}$ \\
\hline $\begin{array}{l}\text { Competencia } \\
\text { de indagación } \\
\text { científica }\end{array}$ & $\begin{array}{l}\text { Control } \\
\text { Experimental }\end{array}$ & $\begin{array}{l}10,29 \\
12,19\end{array}$ & 11,31 & 21 & 13,04 & 40 & $-1,71$ & 0,10 & 2,02 \\
\hline $\begin{array}{l}\text { Capacidad para } \\
\text { problematizar } \\
\text { situaciones }\end{array}$ & Experimental & 11,90 & 46,19 & 21 & 58,10 & 40 & $-0,81$ & 0,42 & 2,02 \\
\hline $\begin{array}{l}\text { Capacidad para } \\
\text { diseñar } \\
\text { estrategias }\end{array}$ & Experimental & 13,33 & 53,33 & 21 & 39,76 & 40 & $-0,73$ & 0,47 & 2,02 \\
\hline
\end{tabular}


para hacer una

indagación

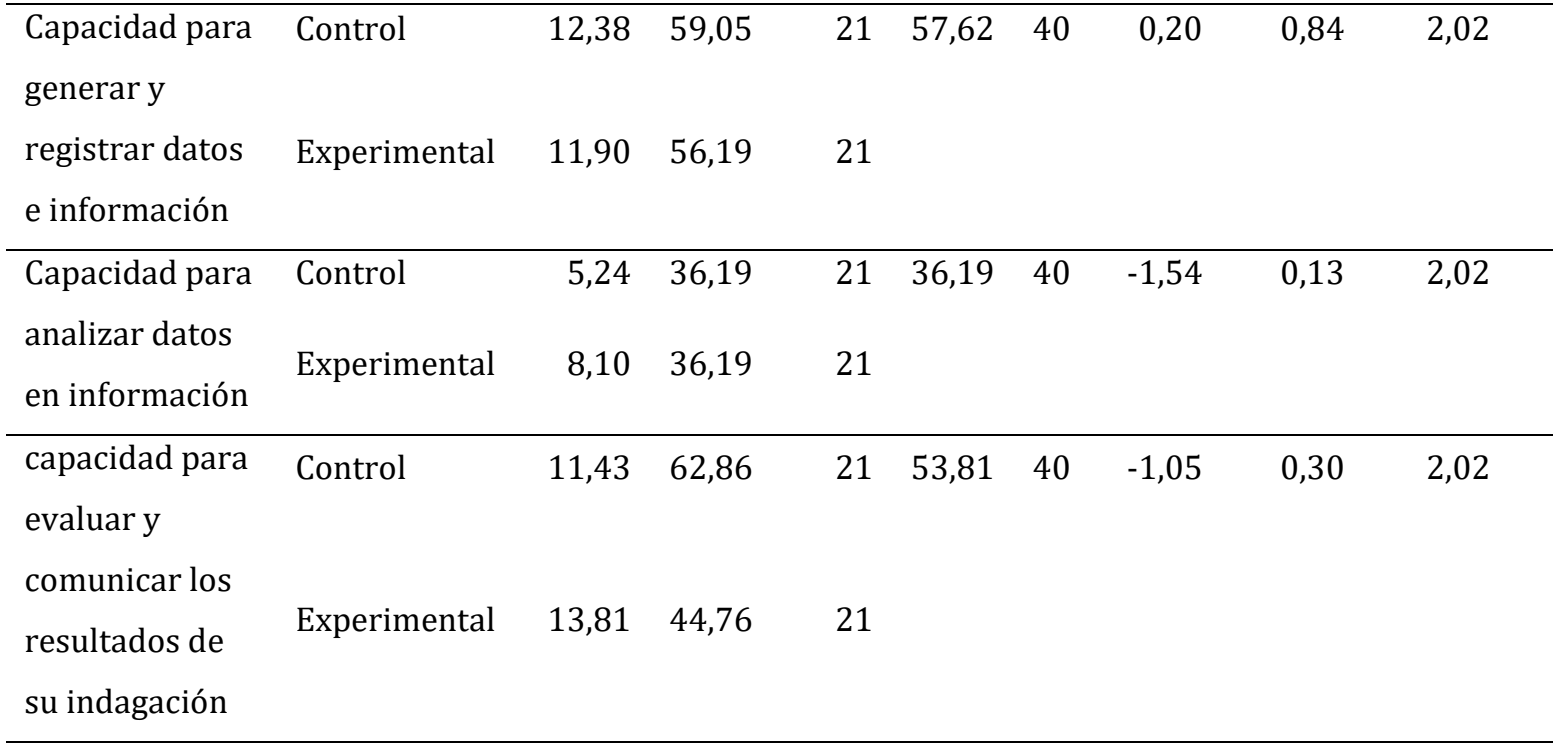

De acuerdo con los datos mostrados (Tabla 4) el p-valor no son significativos para ninguna de las dimensiones. Por lo tanto, con una confiabilidad del $95 \%$, se concluye que las diferencias entre los estudiantes que fueron sometidos al módulo y evaluados mediante las rubricas no significativas.

\section{Discusión}

De acuerdo con los resultados obtenidos tanto en el grupo experimental como en el grupo control estos no son significativos, lo cual contradicen resultados de otros estudios (Martínez, Tellado y Raposo, 2012; Raposo y Martínez, 2014; Urias, Rodríguez \& Zárate 2018); quienes mencionan que la rúbrica es un instrumento que coadyuva en aprendizaje de competencias por parte de los estudiantes y que los que han sido evaluados mediante estos instrumentos están satisfechos con su uso. Sin embargo, la falta de resultados positivos o significativos en este estudio se debería a la extensión en su elaboración de la rúbrica. Cano (2015) señala que la elaboración de las rúbricas debe seguir unos niveles de gradaciones de calidad y tipificando los estándares de desempeño que se quiere medir.

En consecuencia, el problema sobre los resultados de este estudio no estaría en que, los estudiantes no desarrollan la competencia de indagación, sino, en el instrumento de evaluación utilizado, debido a que los descriptores fueron muy amplios, que posiblemente generó falta de comprensión y desinterés por parte del estudiante, que ocasionó que no sea un instrumento que coadyuve al aprendizaje. En este caso es que la posición que se toma frente a esto es que no existen, tal como sugiere Escudero (2010) instrumentos malos o buenos, sino, coherentes o no con 
los resultados de aprendizajes de los estudiantes. Lo cual, desde el punto de vista técnico no estarían bien elaborados para valorar las competencias de los estudiantes. Es necesario, por lo tanto, una confección laboriosa que permita diagnosticar de manera adecuada el proceso de enseñanza aprendizaje e intervenir en su mejora (García, Sempere, Marco y De la Sen, 2011).

Sin embargo, dentro de los resultados a nivel de dimensiones hay algunas diferencias significativas en cuanto al desarrollo de algunas de las capacidades de esta competencia por parte de los estudiantes, tales como que registra datos $e$ información y la capacidad para evaluar comunicar los resultados de su indagación. Estos concuerdan con los reportes de Martínez y Raposo (2011), quienes mencionan que, la rúbrica, independiente a cuál sea la naturaleza o temática que se está estudiando o evaluando, éste permite sistematizar y recopilar mejor la información y evidencias de su trabajo. Además, contribuye de manera eficaz obtener mayor entendimiento del propio proceso de aprendizaje (Huaire y Arteta, 2018) y una mayor autonomía de autorregulación de los estudiantes (Martínez, Tellado y Raposo, 2012), los

\section{Referencias}

Acevedo, J., Vázquez, A., Acevedo, P. y Manassero, M. (2005). Evaluación de creencias sobre ciencia, tecnología y sus relaciones mutuas. Revista Iberoamericana de Ciencia, Tecnología y Sociedad CTS, 2(6), 7399. estudiantes se familiarizan con el uso de esta herramienta y se aproximan a la percepción del docente (Raposo y Martínez, 2014) ayuda a fortalecer el proceso formativo y metacognitivo del estudiante (Arteta y Huaire, 2016).

\section{Conclusiones}

La evaluación del aprendizaje de la competencia de indagación científica es uno de los pilares de la educación y específicamente en la educación básica de nuestro contexto, debido a que la indagación es una metodología de enseñanza, son actividades que el estudiante realiza para desarrollar conocimientos y comprensión de la realidad. De manera general se podría decir que es un comportamiento natural de todo ser humano, que lo realiza casi de manera inconsciente para encontrar explicaciones razonables a cerca de un fenómeno u objeto de estudio. En este estudio se evaluó la competencia de indagación científica utilizando como instrumento la rúbrica, el mismo que dio como resultado no significativo, con lo cual se discute la validez científica de dicho instrumento y que presenta algunas incoherencias en su elaboración. http://www.scielo.org.ar/pdf/cts/ v2n6/v2n6a05.pdf

Acevedo, J., Vázquez, A., Manassero, M. y Acevedo, P. (2002). Persistencia de las actitudes y creencias CTS en la profesión docente. Revista Electrónica de Enseñanza de las 
Ciencias, 1(1), 1-27. http://reec.uvigo.es/volumenes/vo lumen1/REEC 1 1 1.pdf

Acevedo, J., Vázquez, A., Manassero, M. y Acevedo, P. (2003). Creencias sobre la tecnología y sus relaciones con la ciencia. Revista Electrónica de Enseñanza de las Ciencias, 2(3), 353376.

http://reec.uvigo.es/volumenes/vo lumen2/REEC 2 3 9.pdf

Ahumada, P. (2005). Hacia una evaluación auténtica del aprendizaje. Paidós.

Arteta Huerta, H. A. y Huaire Inacio, E. J. (2016). Estrategias metacognitivas y concepciones de aprendizaje en estudiantes universitarios. Horizontes de la ciencia, 6(11), 149158.

https://doi.org/10.26490/uncp.ho rizonteciencia.2016.11.236

Bybee, R. (2004). Scientific Inquiry and Science Teaching. En: Flick, L. y Lederman N. (eds.), Scientific inquiry and nature of science: Implications for teaching, learning, and teacher education (pp. 1-14). Kluwer Academic Publishers.

Camacho, H., Casilla, D. \& Finol, M. (2008). La indagación: una estrategia innovadora para el aprendizaje de procesos de investigación. Laurus, 14(26),284-306.

https://www.redalyc.org/articulo. oa?id=761/76111491014

Cano, E. (2015). Las rúbricas como instrumento de evaluación de competencias en educación superior: ¿Uso o abuso? Revista de Curriculum y formación del profesorado, 19(2),267.

Chacón, M. (2003). Calidad y Equidad en la Educación Media. Santiago de Chile: Biblioteca del Congreso Nacional de Chile. Departamento de Estudios, Extensión y Publicaciones. Depesex/Bcn/Serie Informes, Año XIV, 129.

http://www.bcn.cl/bibliodigital/p bcn/informes/estudios pdf inform es/nro129.pdf.

Cristobal y García, 2013). La indagación científica para la enseñanza de las ciencias. Horizonte de la Ciencia, 3(5), 99-104.

Díaz Barriga, F. (2005). La evaluación auténtica centrada en el desempeño: Una alternativa para evaluar el aprendizaje y la enseñanza. En F. Díaz-Barriga(eds.). Enseñanza situada: Vínculo entre la escuela y la vida. McGraw Hill. https://liceo53.files.wordpress.co $\mathrm{m} / 2013 / 07 / \mathrm{la}$ evaluacion autentic a centrada en el desempenodc3ad azbarriga.pdf

Escudero, T. (2010). Sin tópicos ni malentendidos: fundamentos $y$ pautas para una práctica evaluadora de calidad en la enseñanza universitaria. ICE de la Universidad de Zaragoza. https://dialnet.unirioja.es/servlet/ $\underline{\text { libro? codigo }=667545}$

García, M., Sempere, J. M., Marco, F. y De la Sen, M. L. (2011). La rúbrica de 
evaluación como herramienta de evaluación formativa y sumativa. Universidad de Alicante. https://web.ua.es/es/ice/jornadas -redes2011/documentos/posters/18444 6.pdf

González, C., Cortéz, M., Bravo, P., Ibaceta, Y., Cuevas, K., Quiñones, P., Maturana, J. \& Abarca, A. (2012). La indagación científica como enfoque pedagógico: estudio sobre las prácticas innovadoras de docentes de ciencia en EM (Región de Valparaíso). Estudios pedagógicos (Valdivia), 38(2), 85-102. http://dx.doi.org/10.4067/S0718$\underline{07052012000200006}$

Hernández, N. E. (2015). Actitudes hacia la ciencia en estudiantes de $4^{\circ}$ grado de secundaria del distrito de San Juan de Lurigancho, Lima [Tesis para Optar el Grado de Magíster en Ciencias de la Educación]. Universidad Peruana Cayetano Heredia - $\quad$ UPCH. http://repositorio.upch.edu.pe/bits tream/handle/upch/93/Actitudes. hacia.la.ciencia.en.estudiantes.de.4 \%C2\%B0.grado.de.secundaria.del.d istrito.de.San.Juan.de.Lurigancho.Li ma.pdf? sequence $=3$ \&isAllowed $=y$

Huaire-Inacio, E. J., \& Arteta-Huerta, H. A. (2018). Diferencias en las concepciones sobre el aprendizaje que adoptan los estudiantes de una universidad privada y una pública de Lima. Revista Latinoamericana de Ciencia Psicológica, 10, http://dx.doi.org/10.5872/psienci $\mathrm{a} / 10.2 .25$

López, P. (2017). Indagación científica para la educación en Ciencias. Un modelo de desarrollo profesional docente. Programa de Indagación Científica para la Educación en Ciencias (ICEC), Mineduc de la Universidad Alberto Hurtado. https://educacion.uahurtado.cl/wp site/wpcontent/uploads/2017/04/definiti vo ICEC 16 04.pdf

Márquez, Á. (2006). La Filosofía de Matthew Lipman y el Pensar Filosófico de los Niños. Centro de Filosofía para Niñas y Niños. Universidad Católica Cecilio Acosta. Maracaibo- Zulia.

Martínez, M.E. y Raposo, M. (2011). La evaluación del estudiante través de la rúbrica. IV Xornada de Innovación Educativa. Vigo: Universidad. http://webs.uvigo.es/xie2011/Vig o/XIE2011-077.pdf.

Martínez, E., Tellado, F. y Raposo, M. (2012). La rúbrica como instrumento para la autoevaluación: un estudio piloto. Revista de Docencia Universitaria,11(2), 373-390.

Martin-Hansen, L. (2002). Defining inquirí. The Science Teacher, 69(2), 34-37,

Mesta, M. C. (2018). Aplicación del enfoque de indagación científica en el proceso de enseñanza. Pontificia Universidad Católica del Perú. http://tesis.pucp.edu.pe/repositori o/handle/20.500.12404/10762 
NRC, National Research Council, (2000). Inquiry and the National Science Education Standards. National Academy Press. https://scholar.google.com/scholar lookup?title $=+$ Inquiry + and + the $+\mathrm{N}$ ational+Science+Education+Standa $\underline{\text { rds\&publication year }=2000}$

Pasmanik, D. y Cerón, R. (2005). Las prácticas pedagógicas en el aula como punto de partida para el análisis del proceso enseñanzaaprendizaje: un estudio de caso en la asignatura de química. Estudios Pedagógicos, 31(2), 71-87. http://dx.doi.org/10.4067/S0718$\underline{07052005000200005}$

Pelcastre, L., Gómez, A. R. \& Zavala, G. (2015). Actitudes hacia la ciencia de estudiantes de educación preuniversitaria del centro de México. Revista Eureka sobre Enseñanza y Divulgación de las Ciencias, 12(3), 475-490. https://www.redalyc.org/articulo. oa?id=920/92041414005
Raposo, M. \& Martínez, Ma․ E. (2014). Evaluación educativa utilizando rúbrica: un desafío para docentes y estudiantes universitarios. Educación y Educadores, 17(3), 499. 513.

https://www.redalyc.org/articulo. oa?id=834/83433781006

Schwab, J. (1960). Enquiry, the science teacher, and the educator. The Science Teacher, 27, 6-11,

Schwab, J. (1966). The teaching of science. Harvard University Press,

Schwab, J. (1978). Science, curriculum and liberal education. Chicago, University of Chicago Press.

Urias, C. M., Rodríguez, C. L., \& Zárate, N. E. (2018). Rúbrica para evaluar presentaciones de casos clínicos: instrumento que orienta la calidad en el desempeño. Acta Universitaria, 28(Online First), 83-100. http://dx.doi.org/10.15174/au.201 8.1689. 